\title{
La mémoire d'un bombardement britannique : Notre-Dame-des-Anges (Morlaix, 1943-2003)
}

\section{Yvon Tranvouez}

\section{(2) OpenEdition}

Édition électronique

URL : http://journals.openedition.org/abpo/1308

DOI : $10.4000 /$ abpo.1308

ISBN : 978-2-7535-1493-5

ISSN : 2108-6443

Éditeur

Presses universitaires de Rennes

Édition imprimée

Date de publication : 20 mars 2004

Pagination : 127-154

ISBN : 978-2-86847-976-1

ISSN : 0399-0826

\section{Référence électronique}

Yvon Tranvouez, «La mémoire d'un bombardement britannique : Notre-Dame-des-Anges (Morlaix 1943-2003) ", Annales de Bretagne et des Pays de l'Ouest [En ligne], 111-1 | 2004, mis en ligne le 20 mars 2006, consulté le 01 mai 2019. URL : http://journals.openedition.org/abpo/1308 ; DOI : 10.4000/ abpo. 1308 


\title{
La mémoire d'un bombardement britannique : Notre-Dame-des-Anges (Morlaix, 1943-2003)
}

\author{
Yvon Tranvouez \\ Professeur d'Histoire contemporaine \\ CRBC - Université de Bretagne Occidentale (Brest)
}

Le vendredi 29 janvier 1943 à 14h 15, par temps clair et ensoleillé, deux vagues de six bombardiers Boston de la Royal Air Force, en formation de combat, escortés de nombreux chasseurs et volant à basse altitude pour déjouer la surveillance radar, surgissent dans le ciel de Morlaix, moins d'une minute après le retentissement de l'alerte aérienne; cherchant à détruire le viaduc, ils larguent quarante-huit bombes, sous le feu de la DCA; deux seulement touchent la cible, dont une arche est gravement endommagée, les autres provoquent des dégâts considérables dans les alentours; on relève soixante-sept morts dans la population civile, parmi lesquels trente-neuf enfants et une institutrice des classes maternelles de l'école Notre-Dame-de-Lourdes, et une cinquantaine de blessés; un avion touché par les batteries allemandes s'abîme en mer avant d'avoir pu rejoindre l'Angleterre. Voilà ce que pourrait être, réduit à la sécheresse des faits et à la brutalité des chiffres, le résumé de l'événement que chacun s'accorde à considérer comme le plus tragique de la Deuxième Guerre mondiale à Morlaix. Je n'ai pas l'intention de revenir sur les détails ni sur les circonstances de ce drame dont Michel Le Bars, qui en fut un témoin direct, s'est fait l'historien scrupuleux, aux propos duquel je ne vois pas ce que j'irais ajouter ${ }^{1}$.

Ce qui m'interroge, en revanche, ce sont les formes singulières de la mémoire qui s'est conservée de cet événement, et en particulier cette chapelle Notre-Dame-des-Anges dans la crypte de laquelle sont enterrés les

1. Michel LE BARS, Morlaix. Le bombardement du 29 janvier 1943. Noël 1943 : une grenade, 60 otages. Juin 1940 : l'arrivée des Allemands, Morlaix, pro manuscripto, 1993, 80 p. Du même : " Morlaix sous les bombes, 29 janvier 1943. "Un pont de trop"? ", dans BoHN, Roland et Le BerRe, Alain (dir.), Chroniques d'hier, tome 2, La vie du Finistère, 1939-1945, Bannalec, Imprimerie Régionale, 1994, p. 297-332. Voir aussi BoHn, Roland, Raids aériens sur la Bretagne durant la Seconde Guerre mondiale, tome 2, Les Ailes de l'espérance, de la souffrance et de la liberté, Bannalec, Imprimerie régionale, 1998, p. 54. 
corps ou les restes des écoliers et de leur maîtresse. Il est en effet sans équivalent que des victimes civiles des bombardements aériens de la guerre aient été ainsi inhumées collectivement dans un monument édifié spécifiquement pour cela et à l'endroit même où elles avaient trouvé la mort. Dans les villes les plus affectées, comme Brest par exemple, les familles se chargeaient de la sépulture de défunts que rien ne rapprochait en effet en dehors de leur infortune simultanée ${ }^{2}$. Même une catastrophe aussi traumatisante que celle de l'explosion de l'abri Sadi-Carnot pendant le siège de Brest, en septembre 1944, n'a pas donné lieu à un ensevelissement in situ : l'abri a été conservé, pour la mémoire des générations futures, mais les dépouilles des trois cent soixante-treize victimes sont ailleurs, pour une part dans un carré aménagé à cet effet au cimetière communal de Kerfautras. Pourtant, paradoxalement, cette solennisation propre à Morlaix n'attire guère l'attention. Le voyageur qui arrive à la gare a peu de chances de visiter une chapelle toute proche mais qu'aucune signalisation n'invite à découvrir et qui se trouve de surcroît au fond d'une impasse, alors que sa curiosité sera plus facilement attirée par la dénomination de la place des Otages, dont la situation en plein centre ville fait qu'on y arrive forcément. Cette relative marginalisation d'un lieu de mémoire original pose question. Elle suggère l'hypothèse d'un souvenir sinon conflictuel, du moins embarrassé, hypothèse qu'il faut vérifier en revenant sur le parcours posthume des victimes. Voyons donc ce que les vivants ont fait de ces morts depuis soixante ans, et essayons de comprendre comment et pourquoi ils l'ont fait ${ }^{3}$.

\section{Une mémoire partielle}

Première constatation : la chapelle Notre-Dame-des-Anges n'est pas dédiée à toutes les victimes du bombardement du 29 janvier 1943, mais aux trente-neuf enfants et à l'institutrice tués dans leur école. Qui se souvient encore, hormis leurs familles, des vingt-sept autres civils morts dans la rue, dans leur champ, dans leur maison ou dans les décombres d'un salon de coiffure de la place Thiers? Ne parlons pas des pertes dans les troupes allemandes d'occupation, dont nul ne se préoccupe, au point que personne ne sait dire leur nombre : "peu ", suppose simplement Michel Le Bars ${ }^{4}$. Cette différence de traitement s'explique aisément : c'est précisément le fait que plus de la moitié des victimes sont des petits garçons et des petites filles de quatre à sept ans qui, d'emblée, paraît horrible. "La mort de tout jeunes enfants a particulièrement consterné l'opinion publique ", observe dès le

2. Voir CAPDEVILA, Luc et Voldman, Danièle, Nos morts. Les sociétés occidentales face aux tués de la guerre (XIXe-XXe siècles), Paris, Payot, 2002.

3. Cette étude doit beaucoup aux travaux analogues menés sur la mémoire du conflit vendéen, en particulier à celle d'Alain GERARD, «Les Lucs, Vendée 1794 : l'histoire d'un trou de mémoire ", reprise dans son recueil D'une Grande Guerre à l'autre : la Vendée, 1793-1914, La Roche-sur-Yon, Centre Vendéen de Recherches Historiques, 2002, p. 137-151.

4. Le BARS, Michel, Morlaix..., op. cit., p. 11. 
lendemain du drame le commissaire de police ${ }^{5}$. C'est vrai sur place, ça ne l'est pas moins ailleurs, il n'est qu'à voir, pour s'en convaincre, la façon dont Pierre Limagne, journaliste à La Croix, dont la rédaction est alors repliée à Limoges, est informé de l'événement. " Aujourd'hui, bombardement du viaduc de Morlaix ", note-t-il dans ses éphémérides à la date du 29 janvier 1943. " À Morlaix, quarante-deux enfants d'une école maternelle auraient été tués et de nombreux autres blessés lors de l'opération contre le viaduc ", ajoute-t-il deux jours plus tard. C'est seulement le 5 février qu'il précise qu'à Morlaix " il y a eu soixante-sept morts ", sans doute sur la foi du bilan dressé le même jour par L'Ouest-Éclair ${ }^{6}$. L'attaque, les enfants tués, les autres victimes : la progression des nouvelles est significative.

Il se trouve que cette séparation symbolique se double immédiatement d'une mise à part réelle qui se manifeste dès les obsèques, le $1^{\text {er }}$ février. Alors que L'Ouest-Éclair assure encore le matin même qu'elles auront lieu " globalement aujourd'hui lundi à 10 heures, à l'église Saint-Matthieu ", ce sont en fait deux cérémonies parallèles qui sont organisées à la même heure, comme l'annonce La Bretagne, dont la rédaction, sur place, a été informée à temps des nouvelles dispositions. La cérémonie officielle se déroule effectivement à Saint-Matthieu, sous la présidence de $\mathrm{M}^{\mathrm{gr}}$ Duparc, venu tout exprès de Quimper malgré son grand âge (quatre-vingt-cinq ans) et son évidente fatigue, devant les autorités civiles et militaires, mais sans les dépouilles des enfants et de leur institutrice. Celles-ci sont en effet à l'église de Saint-Martin, où, en présence de la Supérieure générale des Filles du Saint-Esprit, la congrégation en charge de l'école Notre-Dame-deLourdes, le recteur célèbre la messe d'enterrement de ceux et celles qu'il considère comme ses ouailles, l'école étant située en effet sur le territoire de sa paroisse. Étrange spectacle : dans son allocution à Saint-Matthieu, l'évêque, qui s'était préparé pour des obsèques générales, parle surtout des enfants, auxquels tout le monde pense comme lui, face à des cercueils qui ne sont pas les leurs mais ceux des Morlaisiens ordinaires qui ont été atteints par l'une ou l'autre des bombes qui sont tombées sur la ville.

Michel Le Bars explique cette dissociation tardive par un problème pratique que les pouvoirs publics auraient finalement pris en considération : il fallait répartir l'affluence prévisible, à laquelle une seule église n'aurait pu faire face, d'autant plus que le nombre des catafalques allait forcément réduire considérablement la place disponible. Explication plausible : de fait, d'un côté comme de l'autre, le $1^{\text {er }}$ février, la foule est telle que de nombreuses personnes sont obligées de rester dehors ${ }^{7}$. Mais la vraie raison est tout autre : les autorités se sont heurtées au refus obstiné de l'abbé Etienne

5. Arch. dép. du Finistère, 200W86, rapport du commissaire de police de Morlaix au préfet du Finistère, 30 janvier 1943.

6. Pierre Limagne, Ephémérides de quatre années tragiques, 1940-1944, tome 2, De Stalingrad à Messine, Paris, Bonne Presse, 1946, p. 1012, 1015 et 1029. On sait que ces volumes reproduisent les notes prises au jour le jour pendant l'occupation par l'auteur, journaliste à La Croix.

7. LE BARS, Michel, Morlaix..., op. cit., p. 27-33. 
Monfort de renoncer à ses prérogatives de recteur de Saint-Martin ${ }^{8}$. Au préfet Guillon qui, retenu à Brest, n'a pu participer aux obsèques, le sous-préfet Husson adresse le lendemain un long rapport dans lequel il regrette d'avoir à déplorer la " manifestation déplacée " de cet ecclésiastique " qui confond entêtement imbécile avec autorité et ténacité ", ajoutant "qu'il est lamentable dans un pays qui se dit "catholique" de trouver des prêtres qui refusent d'obéir à leur évêque ", et qu'il veut espérer que $\mathrm{M}^{\mathrm{gr}}$ Duparc démettra le recteur de ses fonctions ${ }^{9}$. Cette irritation se comprend quand on sait que le régime de Vichy prescrit alors aux préfets de veiller à la mise en scène solennelle des funérailles collectives des victimes des bombardements alliés ${ }^{10}$. Le spectacle d'une foule massive stationnée, faute de place, à l'extérieur de l'église Saint-Matthieu aurait assurément mieux servi la propagande gouvernementale. L'affaire laisse des traces puisque, un mois et demi plus tard, l'abbé Monfort étant toujours en poste, le nouveau souspréfet, Pierre Capifali, croit nécessaire de rappeler « l'attitude peu opportune du curé de la paroisse de Saint-Martin, refusant d'admettre que les obsèques des écoliers de Notre-Dame-de-Lourdes aient lieu à la paroisse principale de Saint-Matthieu, comme celles de toutes les autres victimes ${ }^{11}$ ". Husson ou Capifali, ces fonctionnaires de l'État français ne mesurent manifestement pas ce que peut être le degré d'indépendance de fait, sinon de droit, d'un rector potens du Léon. Souvent en conflit avec l'administration diocésaine, parfois brouillé avec ses confrères, l'abbé Monfort ne consentira à démissionner, sur la pression du curé-archiprêtre de Morlaix, qu'en 1954, à l'âge de soixante-seize ans; refusant les nominations qui lui seront alors proposées par l'évêché, il se retirera comme prêtre habitué à SaintJean-du-Doigt et mourra trois ans plus tard. Au total, il n'aura payé son individualisme frondeur que d'une tardive promotion, en 1952 seulement, au canonicat honoraire, et d'un silence nécrologique de La Semaine religieuse.

\section{Un ancrage catholique}

Deuxième constatation : le coup de force du recteur de Saint-Martin isole, parmi les victimes du bombardement, celles qui sont notoirement catholiques. Cela contribue, s'il en était besoin, à entretenir le sentiment que c'est cette partie de la population qui a été le plus durement frappée par le malheur. Dans un espace aussi conflictuel que celui de Morlaix, ville bleue flanquée des campagnes blanches du Léon et des campagnes rouges du Trégor ou de la Montagne, cela n'est pas insignifiant. Or il suffit d'ob-

8. Étienne Monfort (1878-1957) était recteur de la paroisse depuis 1939. Sur les règles en usage pour l'organisation des funérailles, voir les Statuts synodaux publiés par Monseigneur Duparc, Quimper, Imprimerie Cornouaillaise, 1928, p. 44-48.

9. Arch. dép. du Finistère, 200W86, rapport du sous-préfet de Morlaix au préfet du Finistère, 2 février 1943.

10. CapdeVIla, Luc et Voldman, Danièle, op. cit., p. 200.

11. Arch. dép. du Finistère, 200W42, rapport du sous-préfet de Morlaix au préfet du Finistère, 24 mars 1943. 
server, comme le fait le rédacteur d'O Lo Lê, le journal catholique illustré pour les jeunes publié à Landerneau par les frères Caouissin, qu'en dehors du viaduc et d'immeubles appartenant à des particuliers, les bombes ont touché une église, Saint-Melaine, une école chrétienne, Notre-Dame-deLourdes, et un cimetière, Saint-Charles, pour faire apparaître comme une évidence la géographie catholique de la tragédie du 29 janvier ${ }^{12}$.

Ce sont donc des catholiques, ces enfants dont les parents recevront bientôt un message de sympathie du pape ${ }^{13}$, mais précisément ce sont des enfants : l'émotion générale tient à ce que leur mort paraît particulièrement injuste, et là commencent les difficultés des prédicateurs qui prennent la parole lors des obsèques. Le malheur immérité, c'est la croix des théologiens, qui en sont toujours réduits à l'alternative du Livre de Job. Ou bien, à l'image de Job sur son fumier, accepter ce qui arrive et renoncer à comprendre en s'abritant derrière un sentiment abrupt de la transcendance : qui sommes-nous pour demander des comptes à Dieu? Ou bien, comme les amis de Job, consternés par son sort, chercher une explication qui donne du sens à ce qui est trop révoltant pour être accepté. Le samedi 30 janvier, sitôt informé des événements et des scènes de désolation liées à la découverte des corps mutilés ou déchiquetés, le vieil évêque a adressé au recteur de Saint-Martin le message suivant, avec instruction de le lire en chaire à tous les offices du lendemain, veille de l'enterrement :

"Cher Monsieur le Recteur,

J'ai cru entendre les gémissements des mères de Bethléem.

Notre-Seigneur aura accueilli vos petits enfants comme ceux qui périrent à sa naissance. Ils prient au Ciel au milieu de nos larmes. Mais combien leurs familles et leur sainte maîtresse sont à plaindre!

Je vous charge de dire mes condoléances paternelles et respectueuses aux pères et aux mères, aux chères Filles du Saint-Esprit, à toute la paroisse et à la cité tout entière atteinte par ce terrible bombardement.

Nous aurons bu le calice jusqu'à la lie.

Le Bon Dieu attachera à notre malheur un mérite d'expiation et de salut. Je vous bénis tout en vous recommandant la prière et l'espoir dans l'amour de Notre-Seigneur Jésus-Christ et de sa Sainte Mère.

Adolphe, évêque de Quimper et de Léon ${ }^{14}$."

Cette réaction immédiate, dictée de toute évidence par le cour et l'émotion, fixe d'emblée un sens et une image lourds de prolongements. Un sens : d'un mot, en parlant d'expiation, $\mathrm{M}^{\mathrm{gr}}$ Duparc réactive la thématique doloriste qui a fleuri dans les milieux ecclésiastiques au lendemain de la défaite de 1940, déclinant sous diverses formes l'idée d'une infortune liée aux péchés de la France ${ }^{15}$. Une image, à travers l'évocation des mères de

12. "La guerre en Bretagne. Barbares!... ", O Lo Lê, n 95, 7 février 1943.

13. « Le Pape Pie XII, ému de compassion envers vos petits enfants, bénit leurs parents et les prie de bien vouloir déposer sur leur tombe une gerbe de fleurs en son nom ", sans date (manifestement 1943), Arch. paroissiales de Saint-Martin.

14. Arch. paroissiales de Saint-Martin.

15. Duquesne, Jacques, Les Catholiques français sous l'occupation, Paris, Grasset, 1986 [1 $1^{\text {re }}$ édition, 1966]; W. D. Halls, Politics, Society and Christianity in Vichy France, 
Bethléem : celle du massacre des saints Innocents, qui lui vient, remarquons-le bien, en songeant à la douleur des parents. Avec le talent oratoire que chacun lui connaît, l'évêque explicite son propos lors des obsèques. Il rend d'abord hommage à l'ensemble des victimes :

"Mais comment, ajoute-t-il, ne serions-nous pas plus particulièrement émus par le sort de ces petits enfants que la mort est venue cueillir sous le manteau de ces saintes femmes que la religion n'affranchit des biens de ce monde que pour répandre plus largement par leur exemple les leçons de l'Évangile? Aucun témoin ne pourrait jamais oublier la vision dantesque des mères affolées, accourues de tous côtés, fouillant les décombres, à la recherche de leurs petits, et emportant dans leurs bras des dépouilles sanglantes, inanimées ou expirantes. La Bible a noté qu'une voix se fit entendre à Rama, celle de Rachel pleurant ses enfants, et ne voulant pas se consoler, parce qu'ils ne sont plus. L'Évangile a raconté le massacre des saints Innocents. Bethléem entendit alors les lamentations des mères, et le sang des nouveaux-nés inonda toute la contrée. C'est la même scène qui se reproduisait ici, il y a quelques jours.

Pourquoi tant de victimes innocentes? Avec des athées qui ne croient pas à l'existence de Dieu, avec des matérialistes qui bornent leur horizon à celui de la terre, le silence serait de rigueur. Mais je parle à des chrétiens que l'Église a pénétrés de sa doctrine. Ils savent, eux, quel est le prix du sang et quelle en est la valeur rédemptrice, et ils comprendront notre prière aux saints Innocents : permettez, ô fleurs des martyrs, que nous mettions en vous notre confiance; faites que nos épreuves présentes contribuent à sauver notre grande et malheureuse Patrie ${ }^{16}$ !

$\mathrm{La}$ " valeur rédemptrice " de la mort des trente-neuf écoliers de NotreDame-de-Lourdes, l'abbé Monfort n'en doute pas non plus. Il va même plus loin. Alors que $\mathrm{M}^{\mathrm{gr}}$ Duparc conduit à Saint-Matthieu un deuil traditionnel, le recteur de Saint-Martin n'a devant lui que les dépouilles des enfants et celle de leur maîtresse, sœur Saint-Cyr, à qui son statut de religieuse et sa mort brutale au beau milieu d'une leçon de catéchisme confèrent une pureté aussi indiscutable que celle de ses élèves ${ }^{17}$. C'est donc la messe des anges qu'il célèbre.

"Les funérailles furent un triomphe, lit-on dans les Annales de la Communauté locale des Filles du Saint-Esprit. [...] L'autel orné de fleurs blanches, les cercueils tout blancs, le chant du paradis, tout nous trans-

Oxford/Providence (EU), Berg, 1995; FoulLLoux, Étienne, Les Chrétiens français entre crise et libération, 1937-1947, Paris, Seuil, 1997.

16. « Morlaix en deuil », La Semaine Religieuse de Quimper et de Léon, 12 février 1943. Pour les références bibliques, voir Jérémie, 31, 15, et l'évangile selon saint Matthieu, 2, 16-18 : " Alors Hérode, voyant qu'il avait été joué par les mages, fut pris d'une violente fureur et envoya tuer, dans Bethléem et tout son territoire, tous les enfants de moins de deux ans, d'après la date qu'il s'était fait préciser par les mages. Alors s'accomplit l'oracle du prophète Jérémie : Dans Rama s'est fait entendre une voix, qui sanglote et moult se lamente : c'est Rachel qui pleure ses enfants; et ne veut pas qu'on la console, car ils ne sont plus."

17. De son vrai nom Herveline Laurent, la sœur Saint-Cyr était née en 1912 à Plouénan (Finistère). 
portait dans ce séjour bienheureux où il n'y a plus ni pleurs, ni souffrances, mais où l'on jouit avec Dieu d'un bonheur sans mélange et sans fin ${ }^{18}$."

Cette transfiguration immédiate du malheur le plus atroce en bonheur le plus désirable, ce basculement brutal de la terre au ciel, changent ces pauvres victimes d'un drame humain en acteurs privilégiés d'un plan divin. Le recteur va jusqu'à parler de "l'offrande " qu'ils ont faite à Dieu de leur vie et dont il ne doute pas qu'elle " hâte la fin de l'épreuve terrible où se débat le monde désaxé ${ }^{19}$ ". Les religieuses ne sont pas en reste, qui évoquent ces " chers petits anges que le Seigneur était venu cueillir comme des victimes pures, pour expier les fautes d'un monde coupable ${ }^{20} »$.

L'abbé Monfort en rajoute quelques semaines plus tard dans un billet rédigé pour le bulletin de l'Euvre du Chapelet des Enfants, une œuvre de piété largement répandue dans les paroisses de France. Il y explique qu'il ne faut pas voir dans la mort des petits écoliers un coup du destin, mais " la main toujours miséricordieuse du Bon Dieu cueillant pour son paradis ces petites âmes qui ne s'épanouissaient que pour lui ". D'ailleurs, ajoute-t-il,

" la jeune institutrice bretonne, très pieuse, qui dirigeait ces jeunes enfants, avait su faire naître chez eux - qui l'aimaient tous beaucoup - de délicieux sentiments. Certains par exemple, ne voulaient pas grandir pour demeurer de petits anges et ne pas offenser le Bon Dieu; d'autre désiraient mourir sous un bombardement pour aller droit au ciel, y intercéder pour la France ${ }^{21}$ ".

Cette fois il va trop loin et s'attire une lettre amicale mais agacée du Docteur Dujardin, qui est aussi le grand-père d'une des petites victimes :

"Je puis vous confier que votre point de vue, c'est le catholique, c'est vrai, peine les parents qui eux ne pensent pas que le désir de leurs enfants fût de mourir sous un bombardement. Dans la peine, les sentiments paternels et maternels priment ${ }^{22}$."

Il n'empêche que la surenchère religieuse déployée dès les premiers jours pour conjurer la douleur des familles a joué un rôle décisif dans la décision d'ériger, à l'emplacement même des classes détruites, un tombeau collectif et une chapelle commémorative, selon le vœu émis à la fin des obsèques par la Supérieure Générale des Filles du Saint-Esprit. Quelques mots très forts, employés et réemployés ensuite sans précaution, suffisent à nourrir un processus bien connu dans la fabrication des lieux saints : qua-

18. Annales de la Communauté Notre-Dame-de-Lourdes, Morlaix, Archives de la Congrégation des Filles du Saint-Esprit, Saint-Brieuc. Je remercie sœur Louise Miossec, archiviste de la Congrégation, de m'avoir communiqué ce précieux document.

19. Monfort, Étienne, "Paroles adressées à la population de Saint-Martin à l'occasion des obsèques des petites victimes du bombardement du 29 janvier ", feuille volante imprimée, Arch. paroissiales de Saint-Martin.

20. Annales de la Communauté Notre-Dame-de-Lourdes, Morlaix, Archives de la Congrégation des Filles du Saint-Esprit.

21. "Angéliques Victimes ", Circulaire du Chapelet des Enfants, n 16, juillet-août 1943.

22. Arch. paroissiales de Saint-Martin, Docteur Louis Dujardin à l'abbé Étienne Monfort, 7 septembre 1943 . 
lifiés de " martyrs ${ }^{23}$ ", de " nouveaux petits Innocents ${ }^{24}$ " dont le sacrifice a "valeur propitiatoire ${ }^{25}$ ", les enfants et leur maîtresse ont vocation à être inhumés ensemble sur cette terre désormais "sacrée " où leur sang a coulé, dans la crypte d'une " chapelle du souvenir ${ }^{26}$ " qui devrait devenir ellemême un nouveau lieu de " pèlerinage ${ }^{27}$ " pérennisant la mémoire et l'intercession $^{28}$.

\section{Une bavure britannique}

On peut invoquer le destin ou la main de Dieu pour faire face à l'insupportable, mais tout le monde sait bien que ces enfants sont morts à cause d'une bombe larguée par l'aviation anglaise lors d'une opération aussi inutile qu'incompréhensible. Inutile, puisque les trains circulent à nouveau sur le viaduc huit jours après l'attaque, et que trois mois plus tard l'arche endommagée est totalement restaurée. Incompréhensible, dans la mesure où il n'était pas difficile d'apercevoir que le succès du bombardement d'un ouvrage d'art situé en pleine ville et fortement défendu par des batteries anti-aériennes était techniquement aléatoire, et qu'il comportait de toute évidence des risques considérables pour la population civile.

"Objectif militaire certes, mais que voulait-on? s'interroge le sous-préfet dès le 2 février. Isoler Brest et rendre difficile le ravitaillement des bases sous-marines qui paraissent elles au moins inaccessibles aux bombes les plus fortes?

S'il en est ainsi, on ne s'explique pas pourquoi on est allé bombarder un endroit où l'on était certain de faucher des vies humaines alors que, en pleine campagne à quinze kilomètres de là, le viaduc de Guimiliau offrait d'autres avantages, en particulier pour les aviateurs de ne rencontrer aucune DCA. Mais sans doute Morlaix a-t-il payé la réclame qu'on a faite autour de son viaduc et au point de vue répercussion dans le monde, il est certain que le bombardement du viaduc de Guimiliau n'aurait pas résonné et affaibli peut-être le moral des populations comme celui de Morlaix ${ }^{29}$."

Les historiens qui se sont penchés depuis sur la question ne sont pas plus avancés. Dans sa minutieuse étude sur la stratégie du Bomber Command en Bretagne, Roger Huguen estime, comme Michel Le Bars avant lui, que le choix de Morlaix était une " aberration ${ }^{30}$ ». Si celui de Guimiliau

23. Annales de la Communauté Notre-Dame-de-Lourdes, Morlaix, Archives de la Congrégation des Filles du Saint-Esprit.

24. La Colombe, bulletin de la Congrégation des Filles du Saint-Esprit, n 17, 1942-1943, Archives de la Congrégation des Filles du Saint-Esprit, série 3P.

25. "Angéliques victimes ", op. cit.

26. Arch. paroissiales de Saint-Martin, Avis de l'abbé Monfort à ses paroissiens, 8 février 1943.

27. Le Courrier du Finistère, 6 février 1943.

28. Sur ce processus, voir DuPRonT, Alphonse, Du sacré, Paris, Gallimard, 1987.

29. Arch. dép. du Finistère, 200W86, rapport du sous-préfet de Morlaix au préfet du Finistère, 2 février 1943.

30. Huguen, Roger, La Bretagne dans la bataille de l'Atlantique, 1940-1945. La stratégie du Bomber Command appliquée à la Bretagne, Spézet, Coop Breizh, 2003, p. 248. 
semblait trop modeste, le viaduc de La Méaugon, près de Saint-Brieuc offrait une cible isolée d'une importance comparable à celle de l'ouvrage morlaisien. Or rien ne permet d'attribuer cette opération à l'égarement d'une décision précipitée : des tentatives avaient déjà eu lieu en avril et juin 1942, une autre le 26 janvier 1943, toutes contrariées en cours de mission par une météo défavorable; en mars 1943 encore, deux attaques nocturnes ne causeront que quelques dégâts matériels dans une ville désormais partiellement évacuée. Il semble avéré aujourd'hui que le bombardement de Morlaix relève d'une erreur d'appréciation du commandement britannique.

Les contemporains de l'événement n'en sont pas moins persuadés, mais compte tenu des circonstances, ils l'expriment diversement, l'indignation des uns contrastant avec la prudence ou les euphémismes des autres. " Raid terroriste de l'aviation anglo-américaine sur Morlaix ", titre $L a$ Bretagne du $1^{\mathrm{er}}$ février. "Sauvage agression sans excuse de la Royal-AirForce ", lit-on dans $O$ Lo Lê une semaine plus tard ${ }^{31}$. Mais la plupart de ceux qui condamnent l'opération, même avec la plus grande sévérité, évitent d'en nommer les auteurs.

«En face de ces trop nombreux cercueils où reposent tant d'innocents, victimes d'un acte odieux et brutal, nous ne pouvons que flétrir hautement ceux qui l'ont perpétré, déclare le recteur de Saint-Martin lors des obsèques. Le sang et les larmes amères qu'ils ont fait verser à ces petits, les jeunes existences qu'ils ont tranchées dans leur fleur, les cœurs de tant de pères et mères qu'ils ont broyés d'une douleur inexprimable, ils en répondront comme d'un forfait, devant la justice de Dieu ${ }^{32}$."

Au même moment, à Saint-Matthieu, $M^{\text {gr }}$ Duparc préfère s'en prendre aux conditions de la guerre moderne :

"C'est une nouvelle page, rouge de sang, que la barbarie vient d'ajouter au tableau de ses exploits. Jadis les règles de la guerre recommandaient aux belligérants d'avoir des entrailles de charité pour les populations civiles, pour les femmes et les enfants sans défense, pour les malades et les vieillards exposés plus souvent à découvert et plus durement aux dangers de la guerre que ne le sont sur le front les soldats en armes. La guerre "totale" de nos temps modernes n'a pas de ces ménagements."

Dans une France que l'ennemi, dit-il, " assaille de tous les côtés " - précieuse litote pour rappeler l'occupation allemande - l'évêque veut de toute évidence éviter de charger les aviateurs alliés ${ }^{33}$.

Curieusement, les représentants du gouvernement ne sont pas moins prudents. Le 3 février, le colonel Bonhomme, officier d'ordonnance du Maréchal Pétain, venu tout exprès de Vichy assurer les familles des victimes de la sympathie du chef de l'État, se borne à féliciter les sauveteurs

31. "La guerre en Bretagne. Barbares!... ", O Lo Lê, op. cit.

32. Monfort, Étienne, " Paroles adressées... ", op. cit.

33. " Morlaix en deuil ", op. cit. 
et à réconforter les blessés ${ }^{34}$. Cette circonspection des autorités tient à l'anglophilie patente de l'opinion publique locale.

"C'est une colère mal contenue qui remplit les cœurs de tous, écrit le sous-préfet, même de ceux les mieux intentionnés à l'égard des AngloSaxons. Mais les autorités allemandes auraient tort de penser que dans cette population qui leur est hostile, cet acte odieux leur ait ramené beaucoup d'adversaires ${ }^{35}$."

Ainsi, le rejet de l'occupant et la confiance accordée aux alliés sont tels que l'émotion et la compassion ne peuvent s'exprimer qu'avec une certaine retenue. C'est déjà, et ce sera de plus en plus, le principal obstacle à la célébration de la mémoire des enfants et de leur maîtresse : impossible d'évoquer leur souvenir sans risquer de ternir l'image des armées alliées.

\section{Une captation bretonne}

Au lendemain du bombardement, le sous-préfet Husson imagine que les sentiments contradictoires induits par l'événement pourraient profiter finalement au régime du Maréchal.

"Seul peut-être le Gouvernement trouvera son compte, suggère-t-il au préfet, en ce sens que les Bretons commencent à comprendre que dans cette guerre révolutionnaire qui fait trembler le monde sur ses bases, chaque peuple travaille pour lui et ne songe guère à la France. "La France seule", disait naguère $M$. Maurras; je crois, en effet, que ce drame aura fait comprendre à beaucoup que la France est abandonnée et que si elle veut sortir de l'ornière ce n'est que par l'union de tous qu'elle y arrivera ${ }^{36}$."

On ne voit pas que cet espoir ait été comblé. Fin mars 1943, le nouveau sous-préfet, Pierre Capifali, observe avec résignation que la population morlaisienne " fait preuve de partialité dans l'interprétation des nouvelles des divers fronts et d'une grande perméabilité aux informations de source anglo-américaine ${ }^{37}$ ". Au demeurant, l'administration n'a pas vraiment cherché à exploiter la mémoire des enfants, qui fait en revanche l'objet d'une relative captation par le mouvement breton, au moins dans sa mouvance

34. « Au nom du Maréchal, le colonel Bonhomme remit aux enfants des jouets, des images, des pots de confiture et, à chacun, un plumier représentant le bâton du Maréchal. Il offrit à tous les blessés une enveloppe contenant un souvenir et les hommes reçurent chacun des boîtes de cigarettes portant le cachet du Maréchal et la francisque ", $L a$ Bretagne, 4 février 1943.

35. Arch. dép. du Finistère, 200W86, Rapport du sous-préfet de Morlaix au préfet du Finistère, 2 février 1943. Sur l'opinion publique en Bretagne pendant la guerre, voir BOUGEARD, Christian, "La Bretagne et les Bretons face à l'Occupation ", dans BougEARD, Christian (dir.), Bretagne et identités régionales pendant la Seconde Guerre mondiale, Brest, CRBC, 2002, p. 13-29.

36. Arch. dép. du Finistère, 200W86, rapport du sous-préfet de Morlaix au préfet du Finistère, 2 février 1943.

37. Arch. dép. du Finistère, 200W42, rapport du sous-préfet de Morlaix au préfet du Finistère, 24 mars 1943. 
catholique $^{38}$. Dès les premiers jours, on l'a vu, $O$ Lo Lê avait donné le ton. Feiz ha Breiz y revient dans sa livraison de mars-avril 1943, par un article signé de son directeur, le très controversé abbé Perrot, "Lazadeg an Innosanted ". À première vue, le recteur de Scrignac ne fait que démarquer les propos de son évêque, mais, à mieux y regarder, il apparaît qu'il explore toutes les virtualités de la comparaison, ce que celui-ci s'était bien gardé de faire. Évitant d'évoquer Hérode, $\mathrm{M}^{\mathrm{gr}}$ Duparc s'en était tenu au sort tragique des saints Innocents. L'abbé Perrot insiste au contraire sur le massacre, analogue à celui ordonné par " le cruel roi Hérode " : "Crime horrible!" ("Torfed euzus! "), écrit-il, adjurant " ceux qui sont responsables du massacre " d'arrêter de " faire le mal ", mais hélas, conclut-il, " c'est l'EspritMalin qui sème le malheur ${ }^{39}$ ". Ici, la mort des enfants n'est pas renvoyée aux desseins insondables de la Providence, mais attribuée aux manœuvres de Satan. La diabolisation des alliés suggérée par cet article sera portée au passif de l'abbé Perrot et invoquée plus tard par les dirigeants de la Résistance comme l'une des raisons qui les ont conduits à le faire exécuter le 12 décembre $1943^{40}$.

À vrai dire, la récupération de la mémoire des enfants de Morlaix n'est pas tant le fait des éléments vraiment nationalistes du catholicisme breton que celui d'esprits plus modérés, qui s'y intéressent d'ailleurs par le hasard des circonstances. Il se trouve en effet, comme on l'a déjà signalé, que le Docteur Louis Dujardin, de Saint-Renan, celtisant érudit que ses travaux ont amené à tisser un vaste réseau de relations dans les diverses ramifications du mouvement breton, est le grand-père et le parrain de l'une des petites victimes. À la mémoire de sa petite-fille et de ses camarades de classe, il compose une longue complainte en breton, Gwerz Lazadeg Skolidigou Itron Varia Lourd, Montroulez, qu'il publie sous son pseudonyme habituel - Loeiz Lok - dans l'hebdomadaire catholique Le Courrier du Finistère du 5 juin 1943. Le texte file la métaphore des petites colombes massacrées par un cruel épervier, "sous prétexte de faire la guerre ", et qui s'envolent avec la sœur Saint-Cyr tout droit vers le paradis, où la Vierge les accueille :

"N'ho tispartin ket, emezi d'ar vestrez.

Merzeriet a-unan 'vit Dou hag ho pro,

A-unan ho pezo digoll eus ho maro.

38. Sur cette mouvance originale, voir LE SQUER, Francis, Les Espoirs, les efforts et les épreuves du mouvement breton catholique de 1891 à 1945, thèse de théologie catholique, Strasbourg, 1997 (publication en l'état à Lille, Presses universitaires du Septentrion, 1999, collection " Thèses à la carte ").

39. Perrot, Yann-Vari, "Lazadeg an Innosanted. Montroulez 29 genver 1943 ", Feiz ha Breiz, mars-avril 1943. On sait que le Malin est le nom souvent donné au démon dans le Nouveau Testament (voir par exemple Matthieu 13, 19). Je remercie Ronan Calvez, maître de conférences de Celtique à l'Université de Bretagne Occidentale, pour la traduction de cet article important.

40. Tranvouez, Yvon, "Les catholiques et la question bretonne (1940-1944) ", dans BOUGEARD, Christian (dir.), Bretagne et identités régionales pendant la Seconde Guerre mondiale, Brest, CRBC, 2002, p. 285-306. 
It, holl, en-dro d'am Mab; kreskit e gurunenn.

Bezit, gant an Aelez, evurus da viken. "

(" Je ne vous séparerai pas, dit-elle à la maîtresse.

Martyrisés ensemble pour Dieu et pour votre pays,

Ensemble vous serez récompensés pour votre mort.

Allez tous, entourez mon Fils; agrandissez sa couronne.

Soyez avec les Anges, heureux à jamais. ")

Martyrisés ensemble, récompensés ensemble, et dès lors s'impose l'avenir posthume que la Supérieure Générale des Filles du Saint-Esprit avait souhaité pour eux, une inhumation collective dans une chapelle dédiée non à Notre-Dame-de-Pitié, comme l'avait d'abord suggéré l'abbé Monfort, mais à Notre-Dame-des-Anges. La chose est dite dans la deuxième version de la gwerz, qui paraît sous forme de plaquette illustrée en décembre 1943, augmentée de quatre pièces dont Chapel an Aelez:

"E koun hor bugale ni' savo eur chapel,

Eur baradozig koant, war an douar santel

Bet glebiet gant o gwad, a zo gwad hor gwad d'eomp. [...]

Aze, eus hor c'halon, e savo pedennou [...]."

("En souvenir de nos enfants nous élèverons une chapelle,

Un joli petit paradis sur la terre sainte

Arrosée par leur sang, qui est le sang de notre sang. [...]

Là de nos cœurs s'élèveront des prières $[\ldots]^{41}$.")

Le Docteur Dujardin n'a pas ménagé ses efforts pour élaborer cette plaquette, qu'il a voulue " exclusivement en breton ${ }^{42}$ " et conçue dans le but de récolter des fonds pour la construction de la chapelle dont il espère bien qu'elle suscitera " la tradition d'un pèlerinage annuel de tous les enfants de Morlaix $^{43}$ ". Pour l'illustrer, il s'est mis en quête de photographies et il a demandé à son ami Micheau-Vernez un dessin qui figurerait l'envol des colombes vers le ciel sur fond de viaduc endommagé. Sans nouvelles de lui, il s'est finalement adressé à Lionel Heuzé, l'architecte morlaisien que James Bouillé lui a recommandé par ailleurs pour la conception de l'édifice dans un style authentiquement breton ${ }^{44}$. Tous ces noms évoquent un milieu bien connu. À lire les lettres qu'il adresse au recteur de Saint-Martin, on voit comment le Docteur Dujardin tire la mémoire des victimes vers la cause bretonne qui lui tient à cœur. Il y revient sans cesse, ainsi dans une lettre adressée à Lionel Heuzé pour lui dire sa satisfaction devant la première esquisse :

41. Lok, L., Gwerz Lazadeg Skolidigou Itron Varia Lourd, Montroulez, 29 a viz genver 1943, Brest, Imprimerie du Château, 1943, 18 pages (la plaquette est tirée à 1000 exemplaires).

42. Arch. paroissiales de Saint-Martin, Louis Dujardin à Étienne Monfort, 7 septembre 1943.

43. Arch. paroissiales de Saint-Martin, Louis Dujardin à Étienne Monfort, sans date (novembre 1943).

44. Sur ce milieu, voir LE CouEdIc, Daniel, Les Architectes et l'idée bretonne, 1904-1945. D'un renouveau des arts à la renaissance d'une identité, Rennes, Société d'Histoire et d'Archéologie de Bretagne, 1995. 
" J'espère que le tout sera en bonne et belle pierre de Bretagne qui défie les siècles et donne à nos oeuvres d'art son cachet. Quand je vois la gare de Brest en papier mâché je frémis d'horreur. Au pays du Kersanton et de la pierre de Laber! [...] Que tous nos vieux architectes et imagiers bretons vous inspirent et vous donnent courage. Comptez que pour ma part je ne négligerai rien pour qu'il vous soit fourni les éléments indispensables à la réalisation de ce que vous voudriez voir réalisé ${ }^{45}$."

On comprend que le Docteur Dujardin soit fait membre d'honneur de la Société immobilière Notre-Dame-des-Anges dès sa constitution en février 1944. Au recteur, qui l'en informe en tant que secrétaire de la société, il répond qu'il entend bien s'associer activement à l'entreprise, ne seraitce qu'en continuant de solliciter les donateurs qui ont déjà répondu à son appel.

" Les milieux qui s'intéressent aux lettres bretonnes ont été particulièrement sensibles et m'ont promis de renouveler leurs gestes généreux, confie-t-il à l'abbé Monfort. Je suis assez connu de ceux qui s'occupent d'art et d'archéologie en Bretagne pour espérer que M. Heuzé, ayant conçu l'œuvre dans son ensemble, trouvera des concours précieux pour la réalisation de certaines parties. Je dis précieux non seulement au point de vue artistique, mais aussi par la générosité dont ils feront preuve ${ }^{46}$."

Rien d'étonnant, dans ce contexte, à ce que, en mars 1944, le quotidien de Yann Fouéré consacre à Notre-Dame-des-Anges un article louangeur, illustré de dessins, qui entraîne le lecteur dans une visite virtuelle de la " chapelle bretonne, simple et fleurie, petite et basse » imaginée par Lionel Heuzé, avant de poursuivre sur un ton prédicatoire :

"Qu'il nous soit permis de souligner, en ces temps où la dissension est à la mode, combien l'unanimité qui s'est faite autour du projet est une chose rare, précieuse, vrai miracle breton. Conscients du deuil collectif qui les avait frappés, autorités civiles et religieuses, municipalité, comité Saint-Martin ont compris que notre ciel n'est pas fait pour le ciment armé, la tuile et les enduits ocrés; qu'ils soient remerciés pour cette volonté unanime de construire breton, pour cette défense de nos sites, pour cette collaboration aux richesses de la Bretagne de demain. "

La construction d'Itron Varia an Aelez, conclut La Bretagne, constitue un " geste d'espérance " particulièrement fort " alors que le monde croule de toutes parts ${ }^{47}$ ".

\section{De la mémoire au mémorial : une reconfiguration}

Le chantier de Notre-Dame-des-Anges n'est pas encore ouvert lorsque, quelques mois plus tard, le monde achève de crouler ou, plus exactement, commence à se relever, en France tout au moins, à la faveur de l'arrivée

45. Arch. paroissiales de Saint-Martin, Louis Dujardin à Lionel Heuzé, 2 décembre 1943. 46. Arch. paroissiales de Saint-Martin, Louis Dujardin à Étienne Monfort, 28 février 1944. 47. Gigout, G., "Les grandes enquêtes de La Bretagne : Notre-Dame-des-Anges ", La Bretagne, 23 mars 1944. 
des troupes américaines et de la libération du pays. On peut dire pourtant qu'à ce moment la mémoire des enfants de Morlaix est déjà largement élaborée, et chargée de connotations qui ne sont pas vraiment accordées au nouveau cours des choses. Pour le résumer en quelques mots, elle est partielle, puisqu'elle n'englobe pas l'ensemble des victimes du bombardement; elle est catholique, alors que l'attitude de l'Église sous l'occupation est quelque peu controversée; elle rappelle fâcheusement que les armées alliées ont commis des erreurs lourdes de conséquences; enfin elle a pris une teinte résolument bretonne, alors que le mouvement breton est largement délégitimé par les excès de ses éléments les plus extrémistes lors des derniers mois du conflit.

À cela s'ajoute qu'entre temps la mémoire morlaisienne de la guerre s'est alourdie et diversifiée. Michel Le Bars remarque que jusqu'au 29 janvier 1943 la ville n'avait pas encore connu les horreurs de la guerre : comme partout, quelques morts au champ d'honneur en 1940, des soldats prisonniers en Allemagne, mais, dit-il, " peu de nos compatriotes se trouvaient dans les prisons allemandes ou dans les sinistres camps de déportés ${ }^{48}$ ». Il n'en va plus de même ensuite. Des soixante otages retenus par l'armée allemande le 26 décembre 1943, au lendemain de l'attentat à la grenade contre le foyer des soldats, et déportés à Buchenwald quelques jours plus tard, trente-deux ne sont pas revenus. En 1944, c'est la Résistance, de plus en plus engagée dans l'action directe, qui subit les conséquences de la répression allemande, à l'instar du groupe « Justice " des FTPF qui perd plusieurs hommes, fusillés en France ou morts en déportation. Il en résulte que, à la libération, la mémoire bleue des otages et la mémoire rouge des résistants communistes se superposent, en quelque sorte, à la mémoire blanche des écoliers de Notre-Dame-de-Lourdes. Mais l'image est trompeuse en ce sens que les deux premières, qui rappellent, selon le langage convenu, la barbarie nazie, sont facilement mobilisables, alors que la dernière, qu'elle renvoie aux errements de l'état-major britannique, au dolorisme de l'épiscopat ou aux dérives du mouvement breton, est, dans tous les cas, plutôt embarrassante.

Dès lors, tout l'effort des familles et des amis des enfants consiste précisément à sauver leur mémoire en la reconfigurant. Ce réaménagement symbolique s'opère dès la libération et se poursuit jusqu'en 1957, date de l'achèvement de la chapelle au terme de "quatorze ans d'obstination, de ténacité, de luttes contre les difficultés de toutes sortes ${ }^{49}$ " qu'il n'est pas superflu d'évoquer ici. Difficultés financières, d'abord. Il n'est pas si facile de transformer l'émotion en souscription, et le temps, qui a la vertu d'apaiser la première, présente l'inconvénient d'affaiblir la seconde. Dès 1947, l'abbé Monfort note dans son journal qu'il « semble qu'il y ait moins d'empressement qu'au passé ${ }^{50}$ ». La quête mensuelle instaurée dans la paroisse

48. Le BARS, Michel, Morlaix..., op. cit., p. 1.

49. Cressard, Christian, " La chapelle N.-D. des Anges ", Ouest-France, 29 janvier 1957.

50. Arch. de l'Évêché de Quimper, 2P254, boite n 12 , Journal historique de la paroisse de Saint-Martin. 
ne suffit évidemment pas, et le comité de propagande de la Société immobilière Notre-Dame-des-Anges imagine divers moyens d'attirer les dons. Les archives du presbytère conservent le manuscrit d'un sermon-type, manifestement composé dès 1944 par l'abbé Monfort et conçu pour être donné dans les paroisses du diocèse. Il commence par planter le décor d'une école heureuse sous le beau soleil de cet après-midi de janvier 1943, les bambins attentifs à la leçon de catéchisme de leur " dévouée maîtresse ", et soudain le fracas des bombes : «Hélas! mes frères, la tragique et inexprimable vision d'horreur dont nous fûmes alors les témoins impuissants autant que désolés et atterrés. "Simple formule rhétorique, naturellement : suit une évocation détaillée des scènes les plus pénibles qui ont frappé les témoins, les corps mutilés, déchiquetés, les pleurs et les cris de désespoir des parents, mais c'est pour mieux émouvoir et souligner, par contraste, la grandeur, la dignité et le recueillement des obsèques, " tant il est vrai qu'il n'y a que la religion chrétienne pour consoler et adoucir vraiment les chagrins les plus amers, les plus grandes détresses ". Appel est fait alors à la générosité des fidèles pour aider à édifier la chapelle " sobre, solide, portant le cachet et la marque de chez nous ", qui va " recouvrir complètement cette terre d'holocauste, désormais sacrée et digne de tous les respects comme la terre bénite de nos cimetières ". Le prêche se termine - est-ce pour redoubler l'élan en associant le souci des vivants à la mémoire des morts? - sur la nécessité de reconstruire aussi, à côté, les classes maternelles, "car, grâce à Dieu, la peur de l'enfant, cette honte de notre temps, n'empêche pas qu'à Saint-Martin les enfants en bas âge ne soient fort nombreux ${ }^{51}$ ".

Une première tranche de travaux est réalisée en 1947, après accord de l'évêché. Fin octobre, un vitrail commémoratif commandé par le recteur est apposé dans le transept nord de l'église paroissiale, tandis qu'à l'école on achève le caveau destiné à recevoir les dépouilles des enfants et de leur maîtresse. Le transfert des corps fournit l'occasion d'une cérémonie solennelle le $1^{\mathrm{er}}$ février 1948, et l'on en profite pour mettre en vente, au profit de la construction de la chapelle, une série de trois images pieuses qui sont diffusées un peu partout, notamment dans le réseau des écoles catholiques de toute la Bretagne. Les offrandes des particuliers restent malgré tout insuffisantes, et il faut se résoudre à solliciter des fonds publics. Au terme de longues tractations, le projet bénéficie finalement du règlement des dommages de guerre par le ministère de la reconstruction et de l'urbanisme et d'une aide de la ville de Morlaix, votée à l'unanimité par le conseil municipal.

Au printemps 1953, alors que la question financière semble enfin en voie d'être résolue, un problème religieux surgit inopinément : $\mathrm{M}^{\mathrm{gr}}$ Fauvel demande que les plans soient examinés par la commission diocésaine d'art sacré. Il se ravise quelques semaines plus tard, le conseil épiscopal considérant que «l'œuvre projetée étant entreprise par des particuliers ", la com-

51. Arch. paroissiales de Saint-Martin, sermon manuscrit de l'abbé Monfort, sans date (vraisemblablement 1944). 
mission n'a pas à intervenir ${ }^{52}$. Incident mineur mais significatif : depuis le début, Notre-Dame-des-Anges est une affaire privée, fondée sur une conception extensive et théologiquement hasardeuse du martyre, portée à bout de bras par un recteur aussi indépendant qu'entreprenant, et finalement canalisée tant bien que mal par les autorités diocésaines. Cette relative marginalisation ecclésiale explique que le pèlerinage annuel rêvé par le Docteur Dujardin n'ait pas pris corps : la procession de quelque deux mille enfants de Morlaix en mars 1949 lors des trois missions paroissiales simultanées reste sans lendemain ${ }^{53}$.

Pour bien mesurer la complexité du processus, il convient de rappeler aussi le poids du contexte politique et religieux de l'époque, marqué à la fois par la force du résistantialisme ${ }^{54}$ et par le regain de la question laïque autour du problème du subventionnement des écoles catholiques, deux éléments qui, de toute évidence, jouent contre une appropriation consensuelle du souvenir des petits écoliers de Notre-Dame-de-Lourdes. La deuxième tranche de travaux débute malgré tout en 1954, et la chapelle enfin achevée est bénite par $M^{\text {gr }}$ Fauvel le 3 février 1957, en présence de nombreuses personnalités, au premier rang desquelles le préfet du Finistère, M. Chapel, qui se trouve être aussi l'oncle d'une des petites victimes.

Revenons donc aux formes de reconstruction de la mémoire qui s'opèrent pendant toutes ces années. Elles relèvent au fond de deux registres : d'un côté, on gomme certaines interprétations faites pendant la guerre et devenues inopportunes; de l'autre, on invente de nouvelles significations. L'évacuation des choses qui fâchent se remarque surtout à l'oblitération systématique de l'attaque aérienne britannique. C'est net dans les discours, ceux qu'on peut lire dans les journaux et qui évoquent le "drame" ", la " catastrophe ", les " décombres ", plus rarement le bombardement luimême, plus rarement encore les bombardiers, jamais le fait qu'il s'agissait d'avions anglais. Voulant voir dans cette tragédie une " conséquence fatale de la technique moderne ${ }^{55}$ ", $\mathrm{M}^{\text {gr }}$ Fauvel déclare en 1957 : "Ce n'est pas le moment d'ajouter à tant d'émotions, tant de deuils que le temps apaise sans les effacer, de rappeler ce drame. Il demeure présent dans le souvenir de cette journée où trente-neuf enfants et leur maîtresse ont été écrasés ${ }^{56}$. " Ainsi écrasés par la technique moderne, les écoliers de Notre-Damede-Lourdes sont avantageusement déconnectés des polémiques relatives à la Deuxième Guerre mondiale.

52. Arch. paroissiales de Saint-Martin, lettre du vicaire général Hervé à l'abbé Monfort, 2 septembre 1953.

53. Voir "Missions paroissiales. À Saint-Melaine, Morlaix ", La Semaine Religieuse de Quimper et de Léon, 20 mai 1949.

54. Voir l'ouvrage désormais classique de Henry Rousso, Le Syndrome de Vichy, Paris, Seuil, 1987.

55. Allocution de $\mathrm{M}^{\mathrm{gr}}$ Fauvel lors de la cérémonie de bénédiction de la chapelle NotreDame-des-Anges, d'après Le Télégramme de Brest et de l'Ouest, 4 février 1957.

56 . Allocution de $\mathrm{M}^{\mathrm{gr}}$ Fauvel lors de la cérémonie de bénédiction de la chapelle NotreDame-des-Anges, d'après Ouest-France, 4 février 1957. 
Cette euphémisation du bombardement britannique est encore plus frappante dans les images. Sur le dessin ornant l'article de l'abbé Perrot dans Feiz ha Breiz en 1943 (fig. 1), on voit des avions noirs s'éloignant d'un paysage dévasté, recouvert d'une épaisse fumée noire et jonché de petits cadavres et de débris de l'école maternelle. Dans la plaquette du Docteur Dujardin, l'envol des colombes composé par Lionel Heuzé (fig. 2) se fait sur fond de viaduc partiellement détruit et la fumée, blanche cette fois, évoque encore l'explosion des bombes. Après la guerre, la guerre, précisément, disparaît. Le vitrail de l'église paroissiale (fig. 3) est tout entier céleste : devant la croix d'où rayonne le Saint-Esprit, la Vierge, Mater misericordiae, accueille la sœur Saint-Cyr et les enfants, en tenue d'écoliers, qui arrivent au paradis. Seules les palmes vertes, symbole du martyre, rappellent « le sanglant holocauste des petites victimes " dont les noms sont inscrits de part et d'autre de la scène centrale ${ }^{57}$. Le message est encore plus aseptisé dans les trois images pieuses dessinées par une religieuses morlaisienne et imprimées en 1948 (fig. 4). Sans doute en est-il une qui représente le viaduc endommagé - très légèrement d'ailleurs - mais la tonalité générale est enfantine et bucolique tout à la fois. On y voit " ces petits êtres se dématérialisant peu à peu pour devenir de blanches petites âmes pures comme des lys ", assure l'imprimeur, qui suggère donc d'atténuer le bleu foncé initialement prévu pour le fond de l'envol des colombes et de choisir un bord jaune " comme le manteau de la Vierge ou bleu pâle comme dans les autres images ${ }^{58}$ ".

Dans ce mouvement qui tend à focaliser l'attention sur le destin céleste des enfants, la comparaison évangélique hasardée par $\mathrm{M}^{\mathrm{gr}}$ Duparc finit par disparaître et on lui substitue une interprétation plus positive du drame du 29 janvier 1943. "Plutôt que de s'indigner devant le scandale de ce nouveau massacre des saints Innocents, écrit en 1957 le chroniqueur du Courrier du Léon, la foi de la population morlaisienne aimera voir en eux des intercesseurs tout-puissants, protégés par le grand manteau bleu de Notre-Dame-des-Anges ${ }^{59}$. " L'idée que dès 1944 ils ont protégé la ville de Morlaix, est explicitement avancée par l'abbé Monfort au terme de la cérémonie du $1^{\text {er }}$ février 1948 :

"Ces petits ne vous semblent-il pas avoir été, en quelque sorte, un élément important de notre rançon, les artisans invisibles, mais non moins efficaces, de notre liberté d'aujourd'hui?

Aux jours angoissants de la libération de notre cité, alors que les pires menaces de l'occupant demeuraient suspendues sur nos têtes, visant à faire de Morlaix une nouvelle ville martyre de notre sol français, qui pourrait ne

57. Allocution de l'abbé Monfort à l'occasion de la bénédiction du vitrail, 28 octobre 1947, dans " À la mémoire de nos chers petits Anges ", document dactylographié composé au lendemain de la cérémonie du transfert des corps le $1^{\text {er }}$ février 1948, Arch. paroissiales de Saint-Martin.

58. Arch. paroissiales de Saint-Martin, lettre de l'imprimeur Danguin à M. Quitté, 20 octobre 1947.

59. «S. Exc. M ${ }^{\text {gr }}$ Fauvel s'est incliné devant les gracieuses victimes du 29 janvier 1943 et a ouvert au culte la Chapelle N.-D. des Anges ", Le Courrier du Léon et du Tréguier, 9 février 1957. 
pas envisager aujourd'hui que le sacrifice total de ces vies innocentes en fleur ne soit pas alors entré en compte aux regards de la justice éternelle et n'ait pas fait pencher, à cette heure critique de notre existence, la balance en notre faveur?

Ne serait-ce là qu'une pieuse illusion ${ }^{60}$ ?"

En somme, le recteur de Saint-Martin suggère que c'est à l'intercession des enfants que Morlaix doit d'avoir connu une libération sans histoire, qui contraste avec le drame des otages de Saint-Pol-de-Léon et la destruction de Brest dans les combats du siège ${ }^{61}$.

L'autre façon de recycler la mémoire des écoliers de Notre-Dame-deLourdes consiste à les inclure dans une mémoire globale des victimes de la guerre. Lors du transfert des corps en 1948, le cortège se dirige de l'église paroissiale à l'école "drapeaux en tête ", ceux des déportés, des anciens combattants, des maquisards, des résistants, des FFL, des anciens prisonniers de guerre, et la presse relève que le conseiller de la République Hyppolite Masson, figure historique de la SFIO finistérienne, s'est joint aux autres personnalités au sortir de la messe ${ }^{62}$. Une messe pendant laquelle le Père Déas, salésien, revenant sur cette " catastrophe navrante " qui a consterné la France entière, esquive habilement les causes secondes dans lesquelles ses prédécesseurs s'étaient enlisés en 1943 et s'emploie à intégrer le souvenir des enfants dans une relecture patriotique des années noires :

« Sort cruel, diront certains! Fatalité impérieuse ajouteront d'autres! Destin inexorable renchériront quelques-uns!

Non pas! Sort, fatalité, destin sont des termes qui n'existent pas dans le vocabulaire chrétien. Un véritable chrétien, en effet, si douloureux que soient les agissements de la Providence, même quand il ne les comprend pas, les accepte et s'interdit de les juger grâce aux fermes assises de sa Foi. Hautement résigné, il proclame qu'on ne peut demander à la terre la solution de problèmes qui touchent à l'Éternité. Dans la plus torturante des souffrances il regarde plus haut qu'elle : il sait que la croix n'a qu'un temps et qu'elle recouvre un bonheur sans fin en conduisant à la Lumière.

Des ténèbres et des tristesses de la terre, élevons-nous donc maintenant vers les clartés et les joies du ciel.

En un gracieux envol, suivant leur infatigable entraîneuse, telles des colombes de pureté, à tire d'ailes, ces angéliques victimes se sont échappées du nid familial, emportant là-haut, vers ce magnifique coin bleu, le blanc des vierges et le rouge des martyrs. Précoces messagers, ils sont allés déposer aux pieds de Notre-Dame des Anges, leur patronne, aux pieds de NotreDame de France, les couleurs de leur pays, de cette Gaule dont elle est la Reine : Regnum Galliae, Regnum Mariae!"

60. Allocution de l'abbé Monfort à l'occasion de la bénédiction du caveau, $1^{\text {er }}$ février 1948, dans "À la mémoire de nos chers petits Anges ", op. cit.

61. Grall, Gilles, La Tragédie de l'été 44 à Saint-Pol-de-Léon, Saint-Thonan, Cloitre Imprimeurs, 1996; FLOCH, Henri et LE BerRE, Alain, L'Enfer de Brest. Brest-Presqu'île de Crozon, 25 août-19 septembre 1944, Bayeux, Heimdal, 2001.

62. "Une émouvante journée du souvenir ", Ouest-France, 2 février 1948. 
Cet habillage tricolore des petits écoliers de Notre-Dame-de-Lourdes, crédités de la " puissance d'intercession " et du " surnaturel apport " qu'ils " ont assuré à notre pays ", les soustrait aux inconvénients d'une mémoire étroitement blanche, exclusivement catholique, et les accommode à l'esprit de l'immédiat après-guerre ${ }^{63}$. Il suffira ensuite d'adapter le motif à l'évolution de la conjoncture. Au moment de l'inauguration de la chapelle en février 1957, en pleine guerre froide, trois mois après Suez et Budapest, tandis que la situation se dégrade en Algérie, $M^{\text {gr }}$ Fauvel ne doute pas que les enfants intercèdent efficacement pour sauver la paix ${ }^{64}$.

Des connotations majeures associées à l'événement pendant la guerre, la moins affectée par le nouveau cours des choses est celle qui renvoie à la dimension bretonne, toujours présente, même si mezza voce. Elle transparaît dans le bois gravé par une artiste morlaisienne en 1948 (fig. 5), où religieuses en habit et femmes en coiffe, emblèmes de la piété bretonne, sont représentées de dos, recueillies devant un caveau symboliquement exhaussé, tandis que l'esquisse de la future chapelle apparaît dans les nuées. Celle-ci, dont les plans sont plusieurs fois revus, en raison notamment des limites du budget, demeure d'une architecture résolument néobretonne, et à chaque fois qu'elle est évoquée dans la presse, c'est pour souligner qu'elle sera " bien de chez nous ", réalisée par les entreprises locales avec les matériaux du pays, granit du Huelgoat ou ardoises de l'Arrée. Au moment de l'inauguration, Ouest-France et Le Courrier du Léon signalent le reportage réalisé par le Docteur Dujardin, alors président de la Fédération culturelle Kendalc'h, dans le cadre de l'émission en langue bretonne diffusée par Radio-Quimerc'h.

\section{La mémoire après le mémorial}

Après 1957, tout se passe comme si la pierre tenait lieu de discours, le mémorial de mémoire. Régulièrement fleurie à la Toussaint et au 11 novembre, mais dépourvue de pèlerinage, la chapelle ne sert guère qu'à la messe anniversaire célébrée chaque 29 janvier. Cette cérémonie, qui mobilise surtout les familles et les religieuses de l'école, est rituellement annoncée au prône du dimanche qui la précède. En 1978, le dispositif change : c'est désormais à l'église paroissiale, le dimanche le plus proche du 29 janvier, pendant l'office, qu'on prie « spécialement pour les enfants victimes du bombardement de Morlaix ${ }^{65}$ ». On sent que le zèle fait défaut. L'abbé Monfort avait fait de cette chapelle une affaire personnelle pour

63. Allocution du Père Déas, salésien de Coat-an-Doc'h, à l'occasion de la bénédiction du caveau, $1^{\text {er }}$ février 1948, dans "À la mémoire de nos chers petits Anges ", op. cit. Sur un processus analogue au début du xx ${ }^{\mathrm{e}}$ siècle, voir Cloître, Marie-Thérèse, "Bleu, blanc, rouge. Marie et Marianne d'après La Semaine Religieuse de Quimper et de Léon, 18861914 ", à paraître dans Kreiz.

64. " $\mathrm{M}^{\mathrm{gr}}$ Fauvel a béni hier la chapelle Notre-Dame-des-Anges ", Ouest-France, 4 février 1957.

65. Arch. paroissiales de Saint-Martin, cahier de prônes. 
laquelle il s'était dépensé sans compter, mais tel Moïse mourant au seuil de la Terre promise, il avait dû quitter la paroisse au moment où la construction commençait enfin, et, pour une raison qu'il n'est pas possible de préciser - maladie? amertume? exclusion? - il n'avait pu être présent à l'inauguration, et s'était éteint huit mois après. Ses successeurs ne savent trop que faire de cet héritage encombrant et fâcheusement situé : il se prête mal à une exploitation pastorale dans ces années post-conciliaires où l'école catholique n'a guère les faveurs du clergé d'avant-garde, qui ne voit que par l'Action catholique, sans compter que tout ce qui est breton fait figure de traditionalisme dans le mouvement catholique et que tout ce qui est catholique fait figure d'archaïsme dans le mouvement breton ${ }^{66}$.

Au-delà de ce cercle, somme toute restreint, du souvenir familial et paroissial, il n'y a guère que la presse qui se fait de temps à autre l'écho d'un passé qui s'éloigne inexorablement. En 1963, "vingt ans après ", Michel Le Bars, alors journaliste à Ouest-France, prend l'initiative d'une série d'articles dont le ton se veut résolument celui d'une évocation sereine, à la fois récit et témoignage, accumulant de nombreux détails " vrais " qui contribuent à créer cet " effet de réel » à quoi tend la narration historique ${ }^{67}$. Dans une telle posture, il redevient possible de parler de "massacre » et d'aborder la question des responsabilités. "Maintenant on peut le dire ", écrit Michel Le Bars, la "folle décision de bombarder le viaduc de Morlaix " a été prise dans un bureau d'état-major britannique, non, certes, par volonté de tuer, mais " par manque de réflexion ", et cela, ajoute-t-il, " ne suffit d'ailleurs pas à pardonner totalement une telle action ${ }^{68}$ ". Malgré son caractère ambivalent, mêlant mémoire et histoire, ce bilan fait l'effet d'une clôture. Il faut attendre 1983 pour voir Le Télégramme prendre l'initiative d'un article qui se contente de donner la parole à quelques rescapés et témoins du drame. La mère d'une des petites victimes assure que " les aviateurs ne cherchaient pas tellement à bombarder le viaduc mais voulaient se débarrasser de leurs bombes avant de regagner l'Angleterre ". Cette assertion surprenante, qui transforme un bombardement programmé en erreur involontaire, témoigne d'un travail de deuil qui s'est opéré à un moment où il n'était pas convenable d'incriminer le commandement allié. "Nous ne connaissons pas l'avis des historiens de la dernière guerre à ce sujet ", commente le journaliste, qui suggère ainsi une séparation décisive entre mémoire et histoire, sans pour autant donner une suite sur ce deuxième registre ${ }^{69}$. Deux jours plus tard, en effet, rendant

66. Tranvouez, Yvon, "La Bretagne catholique : du mythe au folklore ", dans MorisSET, Lucie K., Dieudonne, Patrick et Simon, Jean-François (dir.), Réinventer pays et paysages, Brest, CRBC, 2003, p. 57-69. Pour un éclairage sur le clergé de Morlaix dans la période post-conciliaire, voir LOARER, Agnès, Le traditionalisme à la base : les lettres de la Chouanne (Morlaix : 1970-1982), maîtrise d'histoire, Université de Bretagne Occidentale, 1998.

67. BARTHES, Roland, "Le discours de l'histoire " (1967), repris dans Essais critiques, volume IV, Le Bruissement de la langue, Paris, Seuil, 1984, p. 153-166.

68. LE BARs, Michel, "C'était, il y a vingt ans : le 29 janvier 1943 ", série de six articles, Ouest-France, 29 janvier 1963 et jours suivants. Citations : VI, 6 février 1963.

69. "Il y a quarante ans, l'école Notre-Dame-de-Lourdes était écrasée par une bombe ", Le Télégramme de Brest et de l'Ouest, 29 janvier 1983. 
compte de la messe anniversaire célébrée à l'église de Saint-Martin, le même quotidien remarque que l'abbé Loussouarn « n'a pas voulu trop sensibiliser l'assistance " et note que "l'opinion publique morlaisienne semble partagée à propos de ces sortes de commémorations ", les uns trouvant inutile de raviver la peine des familles, les autres estimant qu'il faut pourtant rendre hommage aux disparus pour ne pas les oublier ${ }^{70}$.

En attendant la chapelle elle-même vieillit. Des travaux d'entretien s'imposent, qui dépassent les moyens de l'association de l'école Notre-Damede-Lourdes, qui en est propriétaire depuis que l'édifice lui a été dévolu, dès son achèvement, par la Société immobilière Notre-Dame-des-Anges. En 1985, décision est prise de faire don à la ville d'un monument qui semble avoir naturellement sa place dans le patrimoine morlaisien. Le conseil municipal en accepte le principe, mais un élu communiste demande si l'on pourra y faire des expositions. Inquiètes d'un possible détournement culturel d'un bâtiment religieux et funéraire tout à la fois, les familles se constituent alors en association, sous la présidence de Robert Paugam, père de l'un des trente-neuf enfants et patron de l'entreprise chargée de la construction de la chapelle, afin d'assurer " le maintien de la chapelle Notre-Damedes-Anges à sa destination première ${ }^{71}$ ", notamment en y organisant à nouveau la messe annuelle du souvenir. Finalement la donation est entérinée en avril 1986, avec une clause stipulant que l'édifice restera à la fois lieu de culte et mémorial des victimes du bombardement.

Tout au long de la période qui va de 1963, date du premier bilan historique établi par Michel Le Bars, à 1993, il semble que la mémoire de l'événement n'a cessé de s'affaiblir, au rythme du vieillissement et de la disparition des parents des victimes. Les cérémonies du cinquantenaire marquent un tournant, en ce sens que leur solennité contraste avec la discrétion des précédentes et témoigne d'une volonté de réappropriation de cette mémoire à l'abandon, quitte à nier d'ailleurs qu'elle ait pu être ainsi délaissée. "Morlaix s'est toujours souvenu ", assure Le Télégramme au lendemain d'une commémoration à laquelle les autorités civiles et religieuses prêtent à nouveau leur concours : la messe célébrée à l'église paroissiale est présidée par le vicaire général Michel Péron, et le maire, Arnaud Cazin, prend la parole à Notre-Dame-des-Anges pour rappeler ce bombardement " absurde ", tandis que le petit Herlé Chapel, neuf ans, lit des extraits de la gwerz composée par son arrière-grand-père, le Docteur Dujardin ${ }^{72}$. Le montage d'une exposition retraçant les événements et présentée dans la chapelle, la sensibilisation des élèves du collège Saint-Augustin par leurs professeurs d'histoire et de géographie, autres éléments qui confirment

70. "Une cérémonie discrète à la mémoire des victimes du bombardement de 1943 ", Le Télégramme de Brest et de l'Ouest, 31 janvier 1983.

71. Article 2 des statuts de l'association Notre-Dame-des-Anges, 24 mai 1985, Archives de l'association de l'école Notre-Dame de Lourdes.

72. "Morlaix s'est toujours souvenu ", Le Télégramme de Brest et de l'Ouest, $1^{\mathrm{er}}$ février 1993. 
l'apparition du sentiment d'un devoir de mémoire, qui ne cesse de croître dans les années qui suivent.

Cela ne va pourtant pas de soi, tant sont ancrées les habitudes d'un regard sélectif entretenu par les institutions qui cultivent le souvenir résistant. En 1999, des élèves de terminale BEP comptabilité du lycée de Morlaix participent au concours national de la résistance et de la déportation, dont le thème est le suivant : "Des plaques, des stèles, des monuments évoquent le souvenir des actions de résistance et la mémoire des victimes des persécutions et des répressions de la période de 1940 à 1945. Recherchez et commentez l'histoire de ces femmes, de ces hommes, de ces enfants." L'énoncé est tel qu'on ne peut s'étonner de ne trouver aucune mention de Notre-Dame-des-Anges dans leur travail, alors que les otages et les résistants y sont abondamment évoqués ${ }^{73}$.

Deux éléments entraînent malgré tout l'évolution des esprits. D'une part, une nouvelle historiographie analyse les conflits armés du Xx siècle comme des meurtres de masse qui traduisent la brutalisation des sociétés modernes, et ce déplacement de la problématique amène naturellement à s'interroger sur la signification des bombardements aériens de la Deuxième Guerre mondiale $^{74}$. Même si l'accent est mis, fort logiquement, sur les bombardements de zone, généralisés après la conférence de Casablanca de janvier 1943, c'est aussi l'occasion de revenir sur les bavures des attaques dites de précision, à l'instar de celle opérée sur le viaduc de Morlaix. D'autre part, la résurgence identitaire bretonne attire l'attention sur une chapelle inspirée par l'architecture régionaliste, et les pèlerins de la deuxième édition du Tro Breiz y font halte le 29 juillet 2003.

La réhabilitation de Notre-Dame-des-Anges se profile en effet au seuil du XXI ${ }^{\mathrm{e}}$ siècle, quand ces facteurs externes se trouvent activés par une nouvelle dynamique interne qui s'exprime au sein des Amis de l'école NotreDame-de-Lourdes. Cette association de parents d'élèves, qui avait vocation à s'investir dans l'animation péri-scolaire, se trouve peu à peu détournée de cette mission par les familles des victimes, qui la rejoignent pour faire en sorte qu'elle veille avant tout à la " pérennisation de la mémoire collective " du bombardement de $1943^{75}$. Les Amis de l'école Notre-Dame-deLourdes assurent ainsi un double relais : relais générationnel, pour un réseau de plus en plus vieillissant et dispersé par les hasards de la vie; relais institutionnel, l'association Notre-Dame-des-Anges étant tombée en

73. Les élèves de Terminale BEP comptabilité du Lycée professionnel Tristan-Corbière, Morlaix, Concours scolaire 1999 de la Résistance et de la Déportation, 67 pages, Archives du Lycée Tristan-Corbière, Morlaix.

74. Voir, entre autres, Mosse, George L., La Brutalisation des sociétés européennes, Paris, Hachette, 1999. Sur les bombardements systématiques des villes allemandes, voir FRIEDRICH, Jörg, Der Brand. Deutschland im Bombenkrieg, 1940-1945, Propylaën, 2003.

75. «Hommage aux victimes du bombardement du 29 janvier 1943 ", Le Télégramme de Brest et de l'Ouest, 29 janvier 2001. Cette orientation prioritaire vers le " devoir de mémoire " est confirmée par le président de l'association, Dominique Caraës (témoignage oral, 10 novembre 2003). 
léthargie après le décès de son président. De son côté, la municipalité participe à nouveau aux commémorations annuelles en faisant déposer une gerbe devant la chapelle. La célébration du soixantenaire, en janvier 2003, fournit l'occasion de mettre en scène une mémoire réconciliée de la Deuxième Guerre mondiale. Le vendredi 24 janvier, les écoliers de NotreDame-de-Lourdes déposent quarante roses blanches et quarante bougies devant la chapelle. Le samedi 25 , la place de la gare, à deux-cents mètres de là, est rebaptisée place colonel Rol-Tanguy, en hommage au grand résistant communiste, figure héroïque de la libération de Paris, originaire de Morlaix et récemment décédé. Le dimanche 26, lors de la cérémonie officielle à Notre-Dame-des-Anges, le maire, Michel Le Goff, évoque " le jour le plus noir de l'histoire récente de Morlaix " et en appelle à un avenir de paix dans une Europe unie ${ }^{76}$.

Au moment de refermer ce dossier, j'en viens à me demander si la question qui m'a amené à l'ouvrir était bien la bonne. Faut-il vraiment s'étonner du relatif délaissement de Notre-Dame-des-Anges jusqu'à une date récente? N'est-ce pas plutôt son existence même qui est surprenante? En toute logique, la mémoire de ces enfants aurait dû s'effacer d'autant plus rapidement qu'ils étaient morts pour rien, du mauvais côté de l'histoire, à la suite d'une décision opérationnelle aberrante du commandement britannique. Et sans doute, en effet, leur souvenir se serait-il perdu au-delà du cercle de leurs proches s'ils n'avaient été réunis avec leur maîtresse dans ce caveau commun, dans la crypte de cette chapelle construite pour cela sur le lieu même du drame.

Mais, comme on l'a vu, cela ne s'est pas fait sans mal, tant il y avait de raisons de diluer l'événement dans la déploration générale des malheurs de la guerre. Notre-Dame-des-Anges n'aurait pas vu le jour sans la conjonction, imprévisible $a$ priori, de quatre éléments décisifs : la ténacité de l'abbé Monfort malgré l'évidente tiédeur de l'évêché, le soutien constant de la congrégation des Filles du Saint-Esprit et des écoles catholiques de la région, l'influence sociale de quelques parents faisant partie des notables morlaisiens, l'implication du réseau culturel breton par l'intermédiaire du Docteur Dujardin. Mais ces quatre éléments ont joué ensuite contre la valorisation de la chapelle : le changement de recteur, la double disqualification de l'école catholique par le camp laïque et par le clergé conciliaire, la réputation droitière des notables concernés et le relatif discrédit du mouvement breton ont fait obstacle à l'appropriation symbolique de NotreDame-des-Anges par l'ensemble de la population morlaisienne. Loin de devenir " un joyau de plus pour votre site déjà si pittoresque " et " la vigiliante sentinelle de l'agglomération ", que le Père Déas se plaisait à rêver en 1948, elle est restée un édifice confidentiel au fond d'une impasse ${ }^{77}$. Ce

76. TANGUY, Ronan, « 43 bombes, 80 victimes ", Le Télégramme, 27 janvier 2003 ; F. L. « 60 ans après, le traumatisme est resté ", Ouest-France, 27 janvier 2003.

77. Allocution du Père Déas, op. cit. 
long refoulement d'un des monuments les plus originaux que le souvenir de la Deuxième Guerre mondiale ait produits, montre la complexité du nœud de mémoire à partir duquel il s'est construit, et qui est sans doute en train de se défaire, usé par le temps.

Figure 1 - Dessin paru dans Feiz ha Breiz, mars-avril 1943

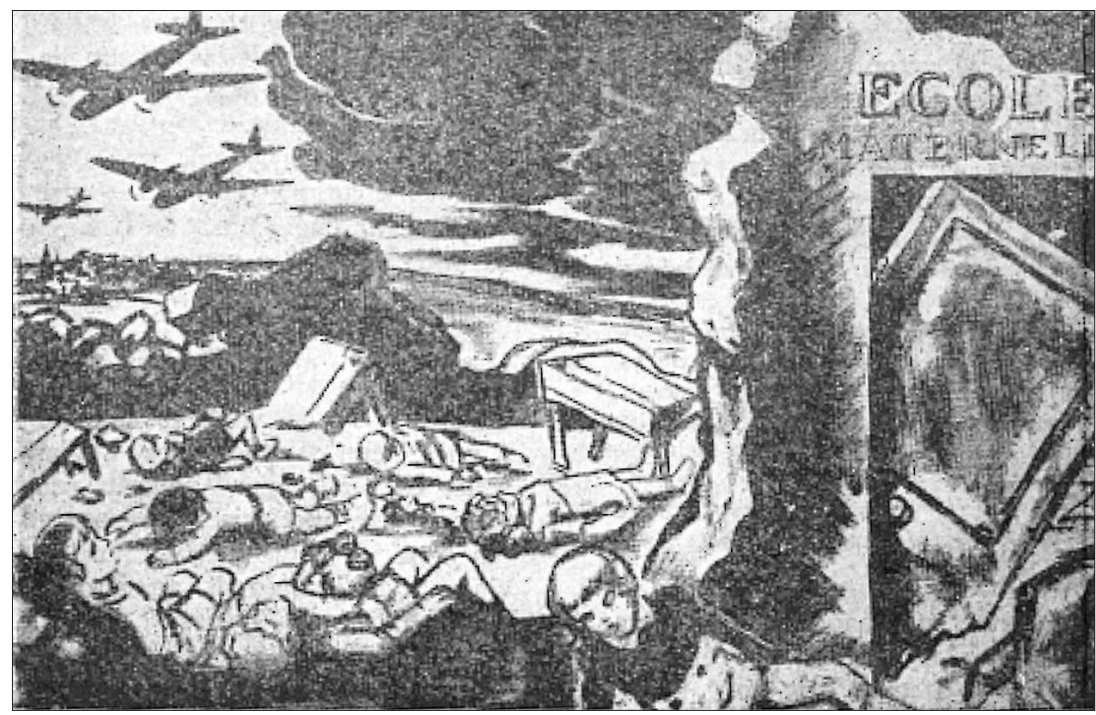



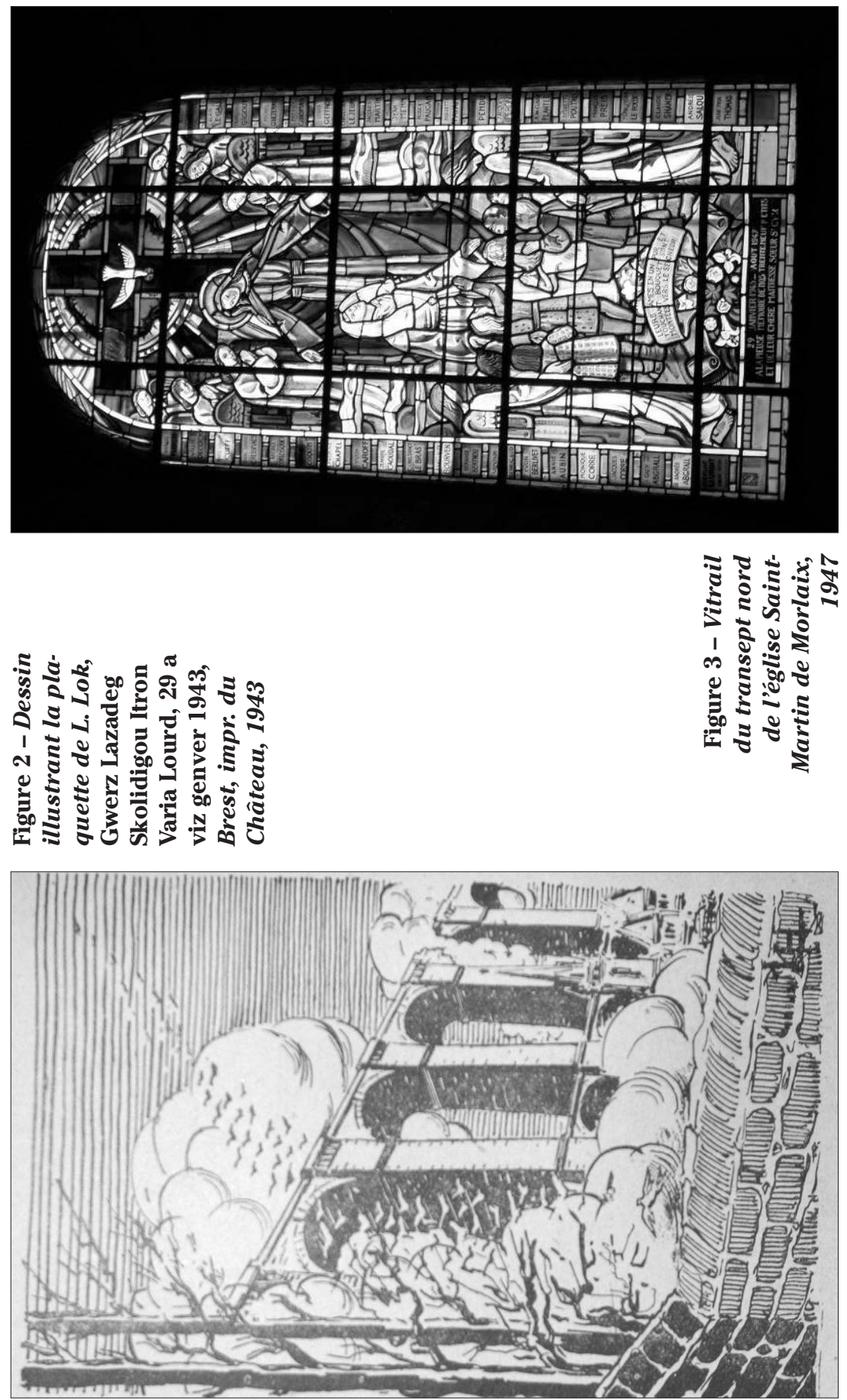


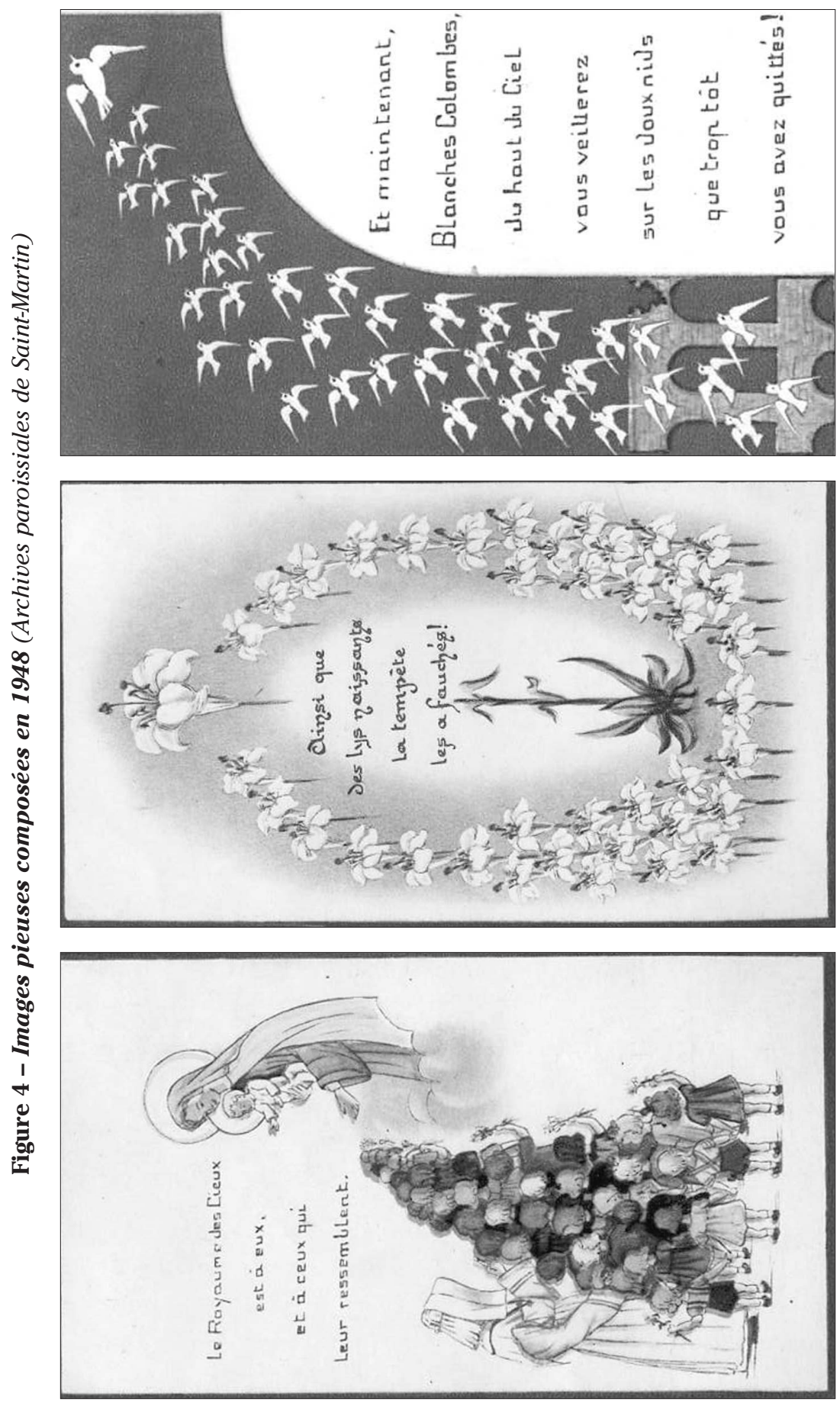


Figure 5 - Bois gravé de F. Herr à l'occasion du transfert des corps, 1948

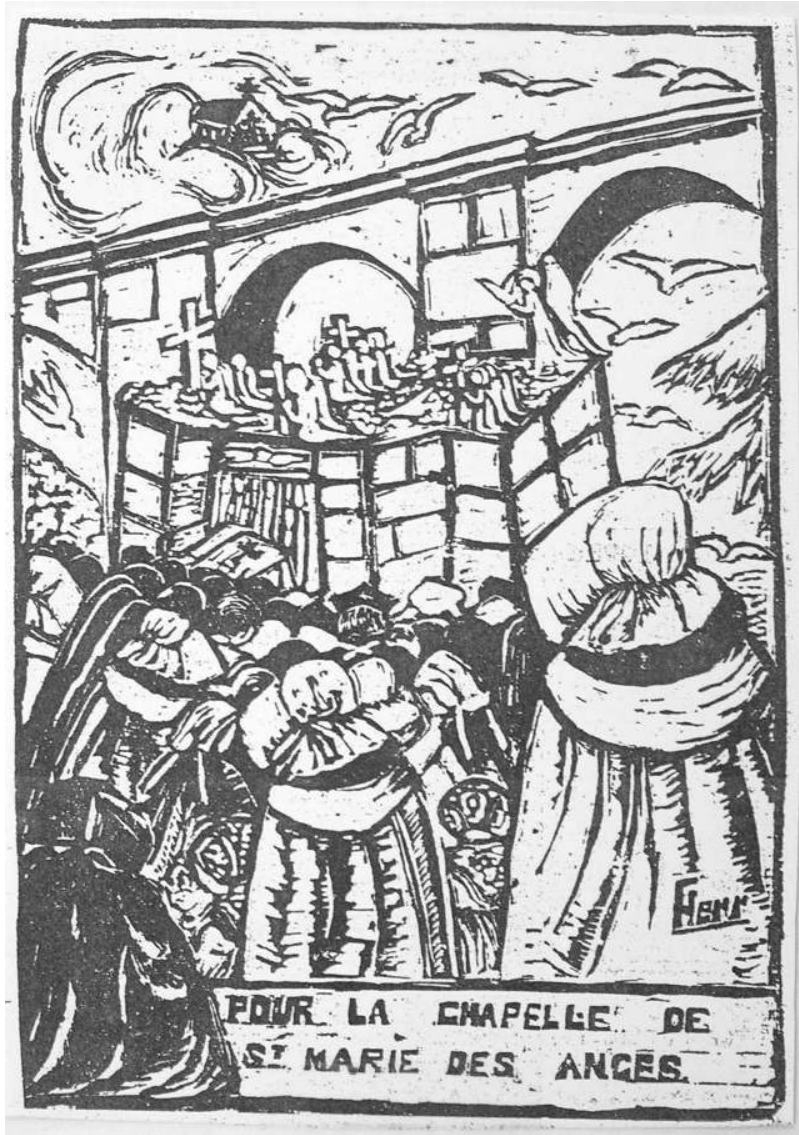




\section{RESUME}

En janvier 1943, un bombardement de l'aviation britannique sur le viaduc de Morlaix fait de nombreuses victimes dans la population civile, parmi lesquelles trente-neuf enfants d'une école maternelle catholique. Dès les obsèques, on observe une volonté de pérennisation de leur souvenir par une future inhumation collective sur les lieux du drame, dans la crypte d'une chapelle édifiée comme mémorial et dédiée à Notre-Dame-des-Anges. Mais ce projet se heurte à divers obstacles qui tiennent notamment à ses connotations particulières, catholique et bretonne, peu propices à une appropriation collective par la population locale, et au fait qu'il attire inévitablement l'attention sur les erreurs du commandement allié pendant la guerre. S’il aboutit malgré tout, en 1957, c'est au prix d'une reconfiguration de la mémoire initiale, réadaptée au nouveau cours des choses. Suit une longue période de quasi oubli, juqu'au moment où, au milieu des années 1990, la valorisation générale du devoir de mémoire et le regard nouveau porté sur la Deuxième Guerre mondiale conduisent à une récupération symbolique de ce lieu désormais investi sur un mode consensuel.

\section{ABSTRACT}

In January 1943, a bombardment of the British aviation on Morlaix's viaduct makes numerous victims in the civil population, among whom thirty-nine children of a catholic nursery school. From their burial, we can see a will of perennisation of their memory by a future collective burial on the scene of the drama, in the crypt of a chapel built as memorial and dedicated to Notre-Dame-des-Anges. But this project collides with different obstacles, especially it's catholic and "Breton" connotations, little convenient to a collective appropriation by the local population, and the fact that it draws inevitably the attention on the errors of the command allied during the war. If it succeeds nevertheless, in 1957, it is for the price of a reconfiguration of the initial memory, readjusted to the new times reality. Follows a long period of almost neglect, until the middle of 1990s, when the general valuation of the duty of memory and the glance on Second World war lead to a symbolic recovery of this place henceforth considered on a consensual mode. 